\title{
Islands of stability in motif distributions of random networks
}

\author{
M.V. Tamm ${ }^{1,4}$, A.B. Shkarin ${ }^{2}$, V.A. Avetisov ${ }^{3,4}$, O.V. Valba ${ }^{4,5,6}$, S.K. Nechaev ${ }^{4,5,7}$ \\ ${ }^{1}$ Physics Department, Moscow State University, 119992, Moscow, Russia \\ ${ }^{2}$ Department of Physics, Yale University, 217 Prospect Street, New Haven, CT 06511, USA \\ ${ }^{3}$ N.N. Semenov Institute of Chemical Physics of the Russian Academy of Sciences, 119991, Moscow, Russia \\ ${ }^{4}$ Department of Applied Mathematics, National Research University Higher School of Economics, 101000, Moscow, Russia \\ ${ }^{5}$ Université Paris-Sud/CNRS, LPTMS, UMR8626, Bât. 100, 91405 Orsay, France \\ ${ }^{6}$ Moscow Institute of Physics and Technology, 141700, Dolgoprudny, Russia \\ ${ }^{7}$ P.N. Lebedev Physical Institute of the Russian Academy of Sciences, 119991, Moscow, Russia
}

\begin{abstract}
We consider random non-directed networks subject to dynamics conserving vertex degrees and study analytically and numerically equilibrium three-vertex motif distributions in the presence of an external field, $h$, coupled to one of the motifs. For small $h$ the numerics is well described by the "chemical kinetics" for the concentrations of motifs based on the law of mass action. For larger $h$ a transition into some trapped motif state occurs in Erdős-Rényi networks. We explain the existence of the transition by employing the notion of the entropy of the motif distribution and describe it in terms of a phenomenological Landau-type theory with a non-zero cubic term. A localization transition should always occur if the entropy function is non-convex. We conjecture that this phenomenon is the origin of the motifs' pattern formation in real evolutionary networks.
\end{abstract}

PACS numbers: 05.70.Fh, 64.60.aq, 80.75.Hc

Studying of complex networks constitutes a rapidly developing interdisciplinary area [1, 2], which unites investigation of various types of experimentally observed biological [3], social [4 [6] and engineering [7] networks, as well as artificial random graphs constructed by various probabilistic techniques $[8-12]$. Many statistical properties of network, including the vertex degree distribution, clustering coefficients, "small world" structure [13] and spectra of adjacency matrices [14] have been extensively treated.

One particular topological characteristic, which seems to be very instructive in providing detailed information about local network topology, is the distribution of small subgraphs (motifs). The presence of motifs of specific type is tightly linked to the network function. For example, the transcriptional regulatory networks have excess of auto-regulation loops with respect to randomly connected graphs with the same vertex degree distribution [15, 16]. Some authors [17] connect the prevalence of specific motif types in the transcription factor networks of E.coili and S.cerevisiae with a network evolution, which might lead to self-consistent optimal circuit design 18 20]. It is known, that protein interaction networks have many short cycles and completely connected subgraphs [21]. This can be thought as a feature necessary for a signal transduction by feed-back loops [18]. These and many other examples clearly demonstrate that the existence of specific motifs in networks strongly correlate with the network function.

The statistical analysis of motifs distribution (MD) in various naturally observed directed networks demonstrates [22, 23] that the simplest three-vertex motifs (triads) of oriented graphs can be split into four broad superfamilies, and the networks within the same superfamily tend to have similar functions. However, to the best of our knowledge, still there is no common opinion about the mechanism behind the separation of MDs into a particular preferred stable state. In this letter we put forward a hypothesis which may give at least a partial answer to this question.

The basic idea of our approach is to notice that a MD gives an essentially coarse-grained description of a network: one integrates out many internal degrees of freedom. Therefore, there exist an entropy corresponding to each particular motif distribution. If some distribution is entropically advantageous, it will occur more often and acts as an effective trap for the network dynamics. Such favorable distributions are the islands of stability in a sea of all MDs, as conjectured in [25].

We consider here statistical properties of simplest motifs - the three-vertex subgraphs or triads - in $N$-vertex random networks. The microscopic configuration of the network is completely defined by $N(N-1) / 2$ Boolean variables denoting presence/absence of edges, while the MD is described by a vector $\mathbf{M}$ whose elements are the numbers of triads $\left\{M_{0}, M_{1}, \ldots\right\}$, or, more conveniently, by vector $\mathbf{c}$ of triad concentrations, $\left\{c_{0}, c_{1}, \ldots\right\}, c_{i}=M_{i} / M$, where $M=N(N-1)(N-2) / 6$ is the total number of triads in the network [42]. For non-directed graphs there are 4 different triad types, shown in the Fig [1, while for directed graphs there are 16, however not all of them are independent (see below).

Studying the entropy of a network as a function of MD from the first principles seems to be an overwhelmingly difficult task. Instead, we model a network in an auxiliary external field, $\mathbf{H}$ coupled to the triad distribution, that is, we consider an ensemble of networks with the partition function $W(\mathbf{H})=\sum_{X}^{\prime} e^{-\mathcal{H}(X) / T}$ with $\mathcal{H}(X)=-\mathbf{H} \mathbf{M}(X)$, where the sum runs over all microscopic configurations $X$ and 'designates conditions im- 
posed on the configurational space (e.g., the conservation of the network degree distribution). Clearly, the unbiased case of equiprobable network configurations corresponds to $\mathbf{H}=\mathbf{0}$. This model is essentially athermic: in the absence of the external field the partition function of the network is purely a combinatorial object with no internal interactions and no temperature dependence (compare this, e.g., with athermic liquid mixtures or non-selfintersecting random walks [26]), it seems therefore natural to introduce normalized dimensionless field $\mathbf{h}=\mathbf{H} / T$, which is the only external variable governing the behavior of the model. This approach, reminiscent of the biased molecular dynamics used, for example, in 27, 28] allows us, by varying $\mathbf{h}$, to skew the motif distribution and thus sample the states of a network which are otherwise unaccessible. As a result, we obtain a full free energy landscape of a network as a function of motif distribution. To equilibrate a network in an external field we permute links as shown in Fig 19, preserving the node degree distribution [24] and use the Metropolis algorithm to accept or reject single steps.

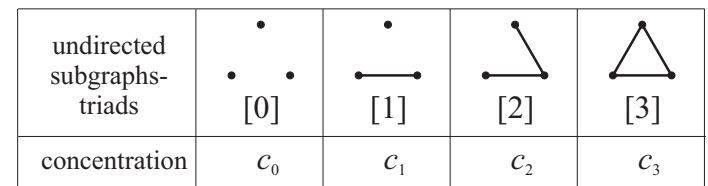

(a)

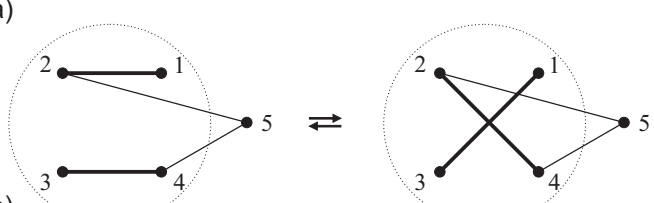

(b)

Figure 1: a) Possible triads in a non-directed network; b) Single link permutation: links (12) and (34) are removed, and links (13) and (24) are created. Triad $\{135\}$ goes from type [0] to type [1], triads $\{125,345\}$ - from type [2] to type [1], and triad $\{245\}$ - from type [2] to type [3]: three new triads of type [1] and one triad of type [3] are created instead of three triads of type [2] and one of type [0], compare to Eq.(1).

For $\mathbf{h}=\mathbf{0}$ the system lives in the largest entropic basin corresponding to some equilibrium distribution of motifs. As $|\mathbf{h}|$ is increased, the motif distribution gets gradually more skewed. In the limit $|\mathbf{h}| \rightarrow \infty$ the entropic effects become irrelevant, and the network approaches the state with the largest possible value of the "energy", i.e. of the product $\mathbf{h} \mathbf{M}$. Depending on the particular shape of the entropy function, the motif vector $\mathbf{M}$ can be either a smooth function of external field $\mathbf{h}$, or it can undergo abrupt jumps at some particular values of $\mathbf{h}$ resembling first-order phase transitions.

Here we look at the simplest case of the triad distribution in undirected networks. The randomization procedure 24] consists of repeated permutations of randomly chosen pairs of links - see Fig 1. Each permutation changes the number of triads of different types: four vertices encircled in Fig 1 belong to $4+6(N-4)+2(N-$
4) $(N-5)$ different triads, of which (i) 4 consist of three vertices constructed from the set (1234), all of them are of type [1] and do not change in the elementary step; (ii) $6(N-4)$ triads including two vertices from (1234) and one external vertex, some of them change as a result of a permutation (we call these changes "elementary reactions" in what follows, not to be confused with "permutation steps" which, generally speaking, consist of many simultaneous elementary reactions), (iii) $2(N-4)(N-5)$ triads including only one of the vertices from the set (1234), they do not change in a permutation. A direct check shows that for an undirected network there is only one possible type of elementary reaction which changes the numbers of the triad types:

$$
[0]+3[2] \rightleftarrows[3]+3[1],
$$

When a new [0]-triplet is formed, it always coincides with formation of 3 triplets of type [2], and elimination of one [3]-triplet and three [1]-triplets (see Fig [1). Equation (1) sets a connection between the time derivatives of the triads concentrations:

$$
3 \dot{M}_{0}(t)=\dot{M}_{2}(t)=-3 \dot{M}_{3}(t)=-\dot{M}_{1}(t),
$$

where $\dot{M}_{i} \equiv \frac{d M_{i}}{d t}(i=0 \ldots 2)$. Since only one triad concentration is independent, the undirected network is effectively $1 \mathrm{D}$ in terms of triads distributions. This means that there exist three independent combinations of $c_{0}, \ldots, c_{3}$, which are conserved under (2), one can choose them as

$$
\begin{aligned}
& I_{1}=\sum_{i=0}^{3} M_{i}=M ; \quad I_{2}=\sum_{i=0}^{3} i M_{i}=3 p M ; \\
& I_{3}=\frac{1}{2}\left(M_{0}+M_{3}\right),
\end{aligned}
$$

$I_{1}$ and $I_{2}$ are, respectively, the total number of triads, $M$, and links, $3 p M$, and $p$ is the fraction of bonds (links) formed in the system. The invariance of $I_{3}$ follows from the conservation of the vertex degree distribution (see the supplementary materials to [22]). We parametrize the one-dimensional evolution of motifs by a variable $m=$ $\frac{1}{2}\left(M_{3}-M_{0}\right)$, or $c=m / M$. The external field $h$ coupled to $m$ is also one-dimensional, and the dimensionless network energy in the external field reads

$$
E=-h m=-\frac{1}{2} h\left(M_{3}-M_{0}\right) .
$$

According to the detailed balance rule, in an equilibrium network subject to energy $E$, for the probabilities of forward, $p_{+}$, and backward, $p_{-}$, permutation steps, one has $p_{+} / p_{-}=e^{-\Delta E}$, where $\Delta E$ is the overall energy change due to all elementary reactions invoked by this permutation step. In the Metropolis algorithm this is achieved by accepting the reaction with probability 1 if it decreases $E$, and with probability $\exp \{-\Delta E\}$ otherwise.

Since each permutation implies many simultaneous elementary reactions (1), the reactions are, generally speaking, not independent. A natural first approximation is, 
nevertheless, to neglect correlations between them and apply the law of mass actions 29, 31] to the chemical kinetics described by Eq.(11). It is straightforward to obtain the implicit $c(h)$-dependence

$$
K \equiv \frac{p_{+}}{p_{-}}=e^{h}=\frac{c_{3} c_{1}^{3}}{c_{0} c_{2}^{3}}=\frac{(A+c)(2-3 p-A+3 c)^{3}}{(A-c)(3 p-1-A-3 c)^{3}},
$$

where $A=I_{3} / M$ (see (3) ), and $K=e^{h}$ is the equilibrium reaction constant $(K=1$ for $h=0)$.

The mean-field equation (5) is applicable to networks with any degree distribution. In what follows we concentrate, though, on a particular example of a conventional Erdős-Rènyi (ER) network [8] with bond formation probability $p$. The triad concentrations in this case are given by:

$$
\begin{aligned}
& \bar{c}_{0}=(1-p)^{3} ; \bar{c}_{1}=3(1-p)^{2} p ; \bar{c}_{2}=3(1-p) p^{2} ; \bar{c}_{3}=p^{3} \\
& \bar{A}=\frac{1}{2}\left(\bar{c}_{0}+\bar{c}_{3}\right)=\bar{I}_{3} / M=\left(p^{3}+(1-p)^{3}\right) / 2
\end{aligned}
$$

All concentrations, as expected, satisfy (5) with $K=1$. Starting with different $p$, we perform a randomization at non-zero fields $h=\ln K>0$. The typical resulting dependences $\phi(h)=c(h)-c(h=0)$ are shown in Fig 2a, b for networks created at $p=0.05$ (a) and $p=0.35$ (b) for various network sizes, $N$. The dashed lines show the $\phi(h)$ dependence as given by solving (5) with $A=\bar{A}$ given be (6). At high $h$ the $\phi(h)$ curve saturates due to depletion of triads of type 2 with growing $c$. In the vicinity of $h=0$ the numerical results are in good agreement with the law of mass action (LMA) (5), while for larger $h$, a sharp change in $\phi(h)$, not predicted by the LMA, is registered. This discrepancy between theory and numerical experiment is, of course, due to the correlations between elementary reactions constituting a single permutation step. To check this, we study 32 the distribution of the number of forward and backward elementary reactions constituting a single permutation step and show that while for small $h$ this distribution is nearly Gaussian (as one expects for independent elementary reactions), in large fields the distribution acquires a peculiar bimodal shape signaling strong correlations between elementary reactions.

For large $p$ the transition between two regimes is very narrow, being, as we shall see, the first-order phase transition. In 32] we show that in this regime a hysteresis typical for first-order transitions is observed. For smaller $p$ the transition is less narrow due to larger fluctuations, although its width decays with increasing $N$. For $p=0.05$ the difference between the specific free energies of two competing phases is relatively small, and both phases could coexist within the whole transition region (compare to the coexistence of two phases in the liquid-liquid 1st order phase transition reported in [30]).

To verify this, we have measured (see [32]) the distribution of links in the network with respect to the number of triangles (type [3] subgraphs) they belong to. The clear

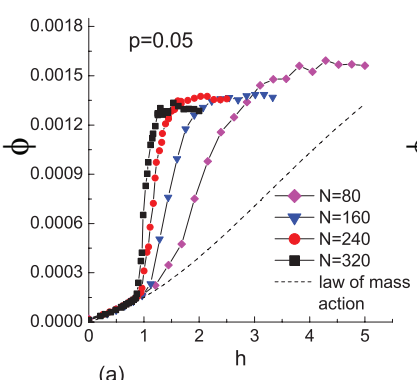

(a)

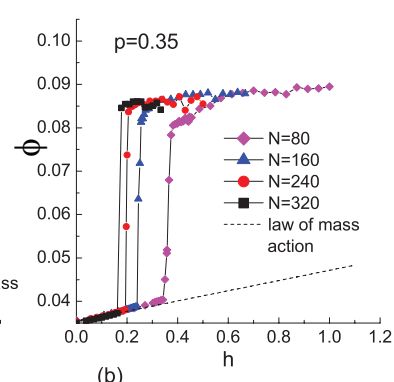

Figure 2: (Color online). The motif distribution $\phi(h)=c-$ $c(h=0)$ in ER networks with $p=0.05$ (a) and $p=0.35$ (b), re-equilibrated at different $h$. Solid lines - numerical results for networks of sizes $N=80$, (magenta diamonds) 160 (blue triangles), 240 (red circles), and 320 (black squares), dashed line - predictions of the law of mass action (5).

bimodality of the distribution allows us to conclude that that there is indeed a phase coexistence in the system: the links in almost triangle-free (low- $h$ ) phase participate only in few triangles, while the links in the triangle-rich (high- $h$ ) phase typically participate in many triangles.

We describe the observed transition via a phenomenological Landau-type theory [31] with $\phi$ as an order parameter. Since there is no $\phi \leftrightarrow-\phi$ symmetry in the problem, the Landau expansion of the free energy is expected to include both odd and even powers of $\phi$, so up to the 4th order term we have

$$
\begin{aligned}
& H=H_{0}-M h \phi ; \\
& H_{0} / M=\frac{\chi}{2} \phi^{2}-\frac{b(N, p)}{3} \phi^{3}+\frac{g(N, p)}{4} \phi^{4}+o\left(\phi^{4}\right)
\end{aligned}
$$

Here $H_{0}$ is a purely combinatorial (i.e., temperature and field-independent) free energy of a network with given motif distribution in the absence of external field, by definition it has a minimum at $\Delta c=0$. The zero-field susceptibility $\chi$, according both to LMA (see (5)) and computer simulations at small $h$, is $N$-independent and can be computed in a straightforward way $\left.\chi \equiv \frac{\partial^{2} H(\Delta c)}{\partial(\Delta c)^{2}}\right|_{h=0}=$

$$
\begin{aligned}
& \left.\frac{\partial h(c)}{\partial c}\right|_{h=0} \text { giving } \\
& \quad \chi=\frac{1}{\bar{c}_{0}}+\frac{9}{\bar{c}_{1}}+\frac{9}{\bar{c}_{2}}+\frac{1}{\bar{c}_{3}}=\frac{1}{p^{3}(1-p)^{3}},
\end{aligned}
$$

where the last equality is true only for ER networks. In turn, higher order coefficients $b(N, p)$ and $g(N, p)$ are expected to be $N$-dependent.

The structure of the Hamiltonian (7) allows for a first order phase transition. Indeed, the equilibrium value, $\phi$, is defined by minimizing $H(\phi)$ in (77), and is given implicitly by equation

$$
\chi \phi-b \phi^{2}+g \phi^{3}=h
$$

For $b^{2}<3 g \chi$ this equation has a single solution for any $h$ (i.e., the free energy $H_{0}$ is always convex), but for 
$b^{2}>3 g \chi$ there exist a region with three solutions, which correspond to two competing minima of the free energy and one unstable maximum in between. In the thermodynamic limit $N \rightarrow$ infty the transition occurs when the values of $H$ match in the minima, while in smaller systems the fluctuations smear the transition.

The numerics for the $\phi(h)$-dependence is well fitted both for $p=0.05$ and $p=0.35$ with the ansatz (9) - see Fig[3. Note that while at $p=0.35$ the transition looks as a discontinuous jump of the order parameter at a point defined by a Maxwell rule, at $p=0.05$ the behavior in the transitional region is given by a linear combination of two competing phases (compare to [30]).

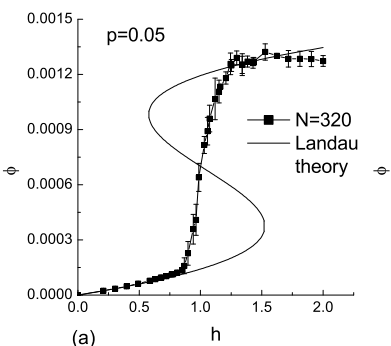

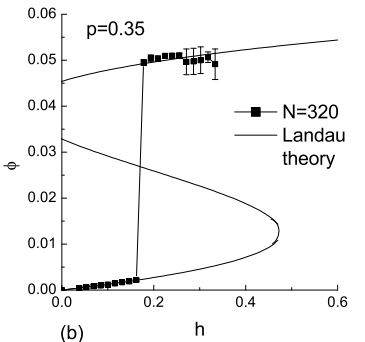

Figure 3: Comparison of the numeric dependence $\phi(h)$ to the solution of the mean-field equation (9) a) numerical results for $N=320, p=0.05$ (squares) and the best fitting Landau theory with $\chi=p^{-3}(1-p)^{-3}=9330, b=1.72 \times 10^{7}, c=8.45 \times$ $10^{9}$; b) numerical results for $N=320, p=0.35$ (squares) and the best fitting Landau theory with $\chi=p^{-3}(1-p)^{-3}=85$, $b=4.46 \times 10^{3}, c=5.7 \times 10^{4}$.

Importantly, the transition points shifts to lower $h$ with increasing size of the network. The detailed analysis of the limiting behavior for $N \rightarrow \infty$ and its impact on the coefficients of the Landau free energy (7) goes beyond the scope of this paper, but se show that the transition 32 value of $h$ seems to decay as $N^{-\alpha}$ with the system size $N$, where $\alpha=0.5 \pm 0.1$. In turn, the width of the transition decays more rapidly with $N$, approximately as $N^{-1}$.

To summarize, we argue that a projection of microscopic (in terms of network nodes) onto macroscopic (in terms of triad concentration) description gives rise to a nontrivial dependence of the entropy on a given motif distribution. In the simplest case of non-directed network considered here, the macroscopic description is effectively one-dimensional. In the presence of an external field, $h$, the equilibrium value of the motif concentration, $\Delta c=c(h)-c(h=0)$, is determined by the balance of energy associated with $h$, and the entropy originating from a mapping of microscopic to macroscopic description. If the entropic landscape is concave, a phase transition into

a state with highly-skewed motif distribution occurs at some critical $h_{\mathrm{c}}$. This transition, observed numerically for ER networks, violates the law of mass action due to correlations between elementary reactions (1) in strong fields.

How general is this transition phenomenon? To check this, we modified the elementary permutation rules allowing an edge connecting two arbitrary vertices $(i, j)$, to be switched to some other pair $(i, k)(k \neq i, j)$. Under this dynamics the nodes degrees are not conserved, and the integral $I_{3}$ in (3) is absent. Accordingly, the dynamics in the motif space becomes effectively two-dimensional with elementary reactions

$$
2[2] \rightleftarrows[1]+[3] ; \quad 2[1] \rightleftarrows[0]+[2]
$$

However, application of an external field $\mathbf{h}$ (which in this case is a 2D-vector) still leads to a transition to a localized motif distribution 33. Another example of a similar transition, which corresponds in the notations of this paper to the dynamics with random creation and elimination of bonds with the external field $\mathbf{h}=0,0, h$ coupled to triangles (the motif parameter space is in this case 3dimensional) was first observed in [34] and discussed theoretical in 35], note also the conjugate effect of bimodality in the triangle parameter estimates in exponentially distributed networks reported in [36]. In [32] we provide preliminary data on networks with fixed scale-free distributions of edge degrees, showing that similar localization transition happens there as well.

The phenomenon of localization of the motif distribution under external field into distinct entropic traps is apparently wide-spread. We conjecture that stable motif profiles constituting superfamilies [22] may correspond to such stability islands inherent to the complicated underlying entropic landscape of a multidimensional motif space of directed networks.

The concept of entropically induced localization may be instrumental in various other fields. Compare it, for example, with the celebrated Eigen model of biological evolution in the space of heteropolymer sequences 37]. There, the localization-delocalization phase transition, known as the "error catastrophe", separates two states, where the genotype is localized in the vicinity of a preferred pattern, and where it is completely random [38 40]. The transition occurs due to an interplay between the attraction to a point-like potential well and the entropic repulsion from this well due to the exponential growth of the number of states with increasing the Hamming distance from the well. In our case, a complimentary phenomenon occurs: the entropic landscape of the system acts as a source of effective attractive traps, while the uniform external field regulates the transitions between trapped states. It seems that trapping of a complex system in stability islands due to a competition between selection and randomness, provides a generic mechanism of localization in complex biological and social systems. 
The authors are grateful to M. Kardar, P. Krapivsky, A. Mikhailov and K. Sneppen for valuable discussions. This work was partially supported by the grunts ANR-2011-BS04-013-01 WALKMAT, FP7PEOPLE-2010-IRSES 269139 DCP-PhysBio, as well as by a MIT-France Seed fund and the Higher School of Economics program for Basic Research.

\section{Supplementary materials}

\section{Appendix A: Distribution of backwards and forwards reactions.}

To study the correlations between elementary reactions we calculated the distribution $P(\mathcal{N})$ of the difference, $\mathcal{N}=\mathcal{N}_{f}-\mathcal{N}_{b}$, between "forward", $\mathcal{N}_{f}$ and "backward", $\mathcal{N}_{b}$, elementary reactions for attempted permutation steps (i.e., regardless of whether it is accepted or rejected) in the skewed ER networks with different fixed values of $\phi$. Note that since in the transition region it is difficult to tune exact value of $\phi$ by choosing external field $h$, we have used a different way of pinpointing a network with given $\phi$. Namely, a network with a desired $\phi=c^{*}-c(0)$ was obtained by equilibrating ER network in a potential $H_{c}=\exp \left(\gamma\left|c-c^{*}\right|^{2}\right)$ with $\gamma$ sufficiently large to make the fluctuations of $\phi$ (or $\left.c^{*}\right)$ negligible, so that the network becomes strongly localized in the state with desired $\phi$. The results of this modelling are presented in the Fig 4
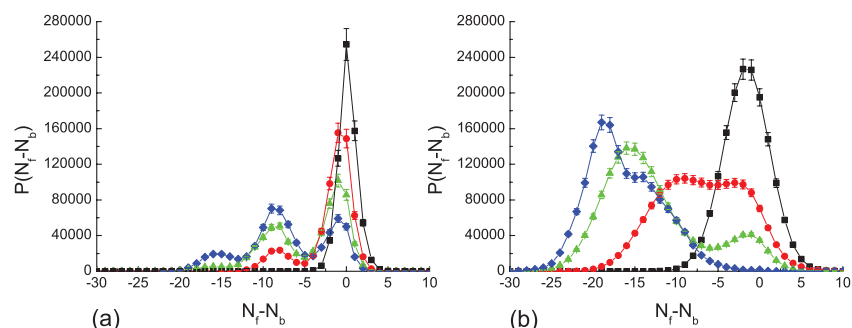

Figure 4: Distribution $P(\mathcal{N})$ for the ER networks with $N=$ 100 and different values of $p$ and $\phi$ : (a) $p=0.05, \phi=10^{-4}$ (black squares), $4.5 \times 10^{-4}$ (red circles), $8.5 \times 10^{-4}$ (green triangles), and $1.2 \times 10^{-3}$ (blue diamonds); (b) $p=0.35$ $\phi=0.001$ (black squares), 0.011 (red circles), 0.028 (green triangles), and 0.034 (blue diamonds). The Y-axis scale is arbitrary.

Close to equilibrium, i.e. at $\phi \approx 0$ the distribution $P(\mathcal{N})$ is shifted to the left, but is still nearly Gaussian, meaning that different backward and forward reactions occur independently and the LMA is still valid. However, as $\phi$ gets progressively larger, the distribution $P(\mathcal{N})$ becomes substantially non-Gaussian, developing a bimodal shape in the transition region $0.01<\Delta c<0.05$. This indicates that the elementary reactions are no longer independent, and all the permutations can be roughly divided into two classes: (i) those which do not change much the motif distribution (the right peak in the distribution), and (ii) those which lead to an essential reduction in the number of triads of type [3], pushing the system towards the equilibrium motif distribution. As $\phi$ approaches the saturation value, the forward reactions $[0]+3[2] \rightarrow[3]+3[1]$ get suppressed, as there are almost no subgraphs of type [2] left in the system, and the backward reactions $[0]+3[2] \leftarrow[3]+3[1]$ become dominant.

\section{Appendix B: Hysteresis in the transition from triangle-poor to triangle-rich phase.}

If the transition from triangle-poor to triangle-rich state is a first order phase transition, one expects a hysteretic behavior of $\phi(h)$. Such a behavior is indeed seen in the simulations for three ER network samples (A, B, C) of size $N=100$ with $p=0.35$ shown in Fig 5 . The hysteresis has been recorded for the $\phi(h)$-dependence when the dimensionless field $h$ is adiabatically increased from zero up to the maximal value $h=2$ and then adiabatically decreased back to $h=0$.

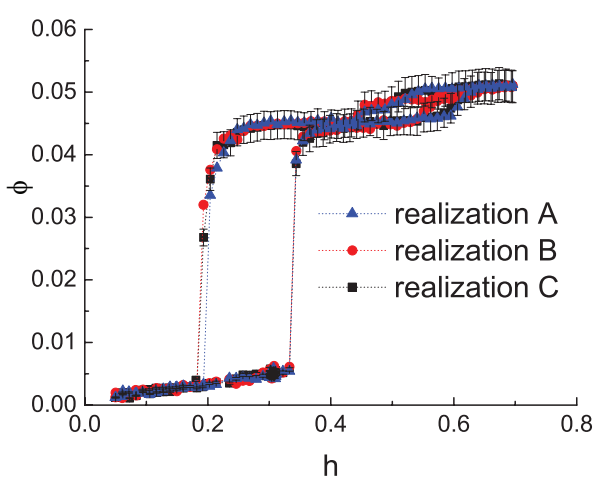

Figure 5: Hysteresis for $\phi(h)$. The curves A, B, C correspond to different samples of adiabatic changes of the field $h$.

\section{Appendix C: Distribution of links with respect to the number of triangles they are involved in}

In order to check that even for the ER networks with small fraction of bonds (e.g. $p=0.05$ ) the transition from triangle-poor to triangle-rich phase is a first-order transition, we have measured the distribution of links in the network with respect to the number of triangles (type [3] subgraphs) they belong to. We expect this measure to correlate strongly with the phase particular link belongs to, therefore, if there is a coexistence of two distinct phases, a bimodality in this destribution is expected. This bimodality is readily observed in Fig 6 allowing us to conclude that that there is indeed a phase coexistence in the system: the links in almost triangle-free (low- $h$ ) phase participate only in few triangles, while the links in 
the triangle-rich (high- $h$ ) phase typically participate in many triangles.

Integrating the first and second "humps" of the curve in Fig 6 one obtains that roughly 2500 links are distributed between phases in proportion 2500:0 at $h=0$, 2200:300 at $h=0.94$ (close to the lower end of the transition region), 1100:1400 at $h=1.19$ (close to the upper end of the transition region), and 400:2100 at $h=1.67$ (above transition region). The latter result may signal either that there is still some left-over triangle-poor phase above the transition region, or, more probably, that the links distribution with respect to the number of triangles in triangle-rich phase is rather wide, leading to some of its links been erroneously identified as belonging to the triangle-poor phase. Thus, for small $(p=0.05)$ and large $(p=0.35)$ density of links, the abrupt change in $\Delta c$ with changing $h$ in ER networks is a first order phase transition from the low- $h$ phase with the MD close to the equilibrium ER network, to the high- $h$ phase with a strongly skewed MD.

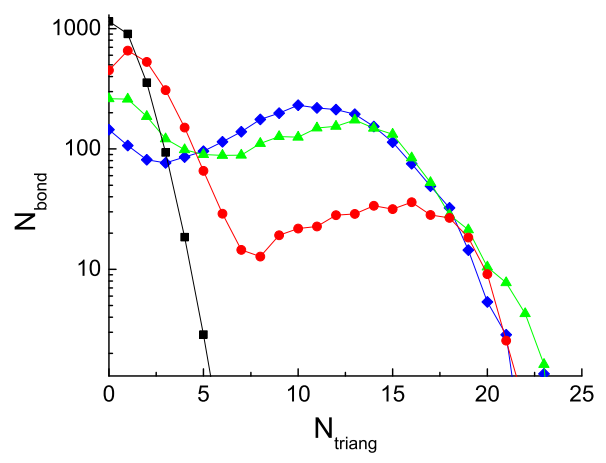

Figure 6: (Color online). Distribution of the links in a network with $N=320, p=0.05$ with respect to the number of triads of type [3] (triangles) they belong to for different values of the auxiliary field $h: h=0$ (black squares), $h=0.94$ (red circles), $h=1.19$ (green triangles, $h=1.67$ (blue diamonds). The bimodality of the curve signals the coexistence of the triangle-rich and triangle-poor phases.

Appendix D: Scaling as $N \rightarrow \infty$.

As it is clearly seen from Figure 2 of the main text, the value $h_{c}$ at which the transition into localized phase occurs decays with growing $N$. In fact, that is to be expected: the total number of motifs in the network $M$ is proportional to $N^{3}$, while the combinatorial free energy (see eq. 7) should probably grow slower (e.g., proportional to the number of links $\sim N^{2}$ ). Fig 7 shows the dependence of the transition point (measured as the point with the largest slope of the $h(c)$ dependence) on $N$ for different values of $p$. The results for both $p$ are compatible with the hypothesis that $h_{c} \sim 1 / \sqrt{N}$, although some divergences from this simple dependence are observed: fitting the log-log plots with straight lines

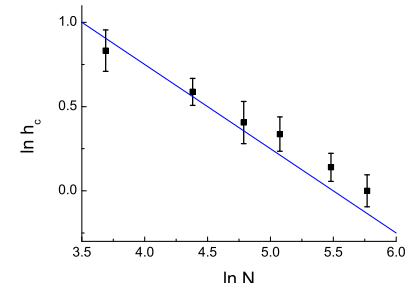

(a)

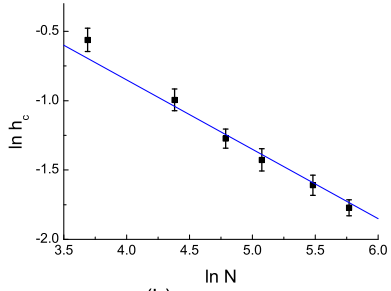

(b)
Figure 7: The $N$ dependence of the transition point as measured by the largest slope of the $\phi(h)$ curve for Erdös-Rényi networks with (a) $p=0.05$ and (b) $p=0.35$. The blue straight lines on both figures have a slope of $-1 / 2$.

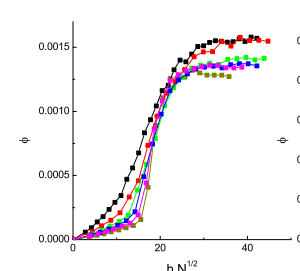

(a)

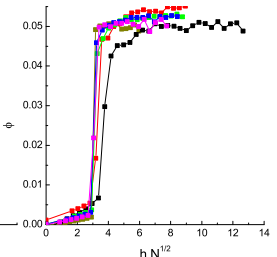

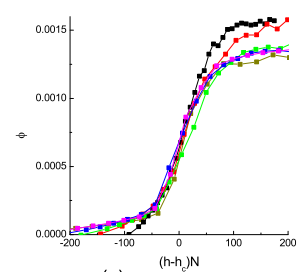

(c)
Figure 8: The $\phi(h)$ dependences in rescaled coordinates for the networks with different $N$. (a), (b) collapse of the transition point in $\phi(h \sqrt{N})$ coordinates for ER networks with $p=0.05$ (a) and $p=0.35$ (b). (c) the vicinity of the transition point for $p=0.05$ plotted in $\phi(h N)$ coordinates. $N=40$ (black), 80 (red), 120 (green), 160 (blue), 240 (magenta) and 320 (dark yellow) for all figures. .

gives the slopes of $\approx 0.43$ and $\approx 0.57$ for $p=0.05$ and $p=0.35$, respectively. As seen from Fig $8(\mathrm{a}),(\mathrm{b})$ the transition points collapse reasonably well when redrawn in $\phi(h \sqrt{N})$ coordinates. Meanwhile, the width of the transition for $p=0.05$ decays roughly as $N^{-1}$ as shown in Fig 8 (c), meaning that even in rescaled coordinates with constant transition point the width of the transition goes to zero with increasing $N$, once again proving that the phenomenon we study is indeed a phase transition (in $p=0.35$ case the transition width is negligible for all network sizes $N$ under consideration).

\section{Appendix E: Scale-free networks in external field.}

To check whether the sharp transition from trianglepoor low- $h$ to triangle-rich high- $h$ state is a general phenomenon, not a peculiarity of Erdős-Rényi networks, we have studied the $\phi(h)$ dependences for the networks with the scale-free node degree distributions of the form $P(k) \sim k^{-\alpha}$. The networks where prepared via a conventional configurational model [41] which is known to give a flat measure over the whole set of networks with a given degree distribution. Networks with $\alpha=2,3$ has been studied, which is close to typical values in experimentally observed scale-free networks, the results are shown in Fig.9 Although the situation is clearly more compli- 
cated than in the ER networks (e.g., the large- $h$ limit of $\phi$ seems to be $N$-dependent while in ER networks it is fully determined by $p$ ), the transition clearly does take place. In the case of $\alpha=3$ there seems to be evidence of an additional intermediate state at $h \sim 0.5$, but this needs further investigation. Note also that since the networks considered here are relatively small, the heavy tail of the $P(k)$ distribution is not sufficiently well-represented. Further, more detailed studies of the localization transition in scale-free networks will be provided elsewhere.
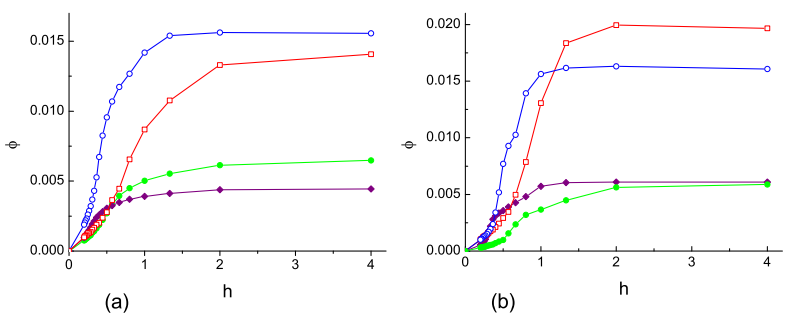

Figure 9: $\phi(h)$ dependence for networks with scale-free node degree distribution $P(k) \sim k^{-\alpha}$, (a) $\alpha=2$, (b) $\alpha=3$. Data for networks with fraction of bonds equal to $p=0.1$ (filled symbols) and $p=0.2$ (open symbols) is shown, network size $N$ is 50 (squares), 100 (circles), and 200 (diamonds).

[1] R. Albert and A.-L. Barabsi, Rev. Mod. Phys., 7448 (2002).

[2] S. N. Dorogovtsev and J. F. F. Mendes, Evolution of Networks: From Biologicals Nets to the Internet and $W W W$ (Oxford University Press, Oxford: 2003).

[3] A.-L. Barabasi and Z. N. Oltvai, Nature, 5, 101 (2004).

[4] J. P. Scott, Social Network Analysis: A Handbook (Sage Publications: 2000).

[5] M. E. J. Newman and J. Park, Phys. Rev E, 68 (036122) (2003).

[6] M.O. Jackson, Social and Economic Networks (Princeton University Press, Princeton, NJ: 2008).

[7] B. Bollobas, Modern Graph Theory (Springer-Verlag, New York: 1998).

[8] P. Erdős and A. Rényi, Publ. Math. Inst. Hungar. Acad. Sci, 517 (1960).

[9] A.-L. Barabasi and R. Albert, Science, 286509 (1999).

[10] P. L. Krapivsky, S. Redner, and F. Leyvraz, Phys. Rev. Lett., 854629 (2000).

[11] S. N. Dorogovtsev, J. F. F. Mendes, and A. N. Samukhin, Phys. Rev. Lett., 854633 (2000).

[12] G. Palla, L. Lovász and T. Vicsek, Proc. Nat. Acad. Sci. USA, 1077640 (2010).

[13] D. J. Watts and S. H. Strogatz, Nature, 393440 (1998).

[14] K.-I. Goh, B. Kahng, and D. Kim, Phys Rev. E, 64 051903 (2001).

[15] G. Balazsi, A. Barabasi, and Z. Oltvai, PNAS, 1027841 (2005).

[16] S. Shen-Orr, R. Milo, S. Mangan, and U. Alon, Nature Genetics, 3164 (2002).

[17] G. Conant, and A. Wagner, Nature Genetics, 34264 (2003).
[18] A. Maayan, S. Jenkins, S. Neves, A. Hasseldine, E. Grace, et al, Science, 3091078 (2005).

[19] M. Csete, and J. Doyle, Science, 2951664 (2002).

[20] S. Mangan, and U. Alon, PNAS, 10011980 (2003).

[21] S. Wuchty, Z. Oltvai, and A. Barabasi, Nature Genetics, 35176 (2003).

[22] R. Milo, S. Itzkovitz, N. Kashtan, R. Levitt, S. Shen-Orr, I. Ayzenshtat, M. Sheffer, and U. Alon, Science 3031538 (2004).

[23] U. Alon, Nature Reviews Genetics, 8451 (2007).

[24] S. Maslov and K. Sneppen, Science, 296910 (2002).

[25] V. A. Avetisov, S. K. Nechaev, and A.B. Shkarin, Physica A 3895895 (2010).

[26] J. des Cloizeaux and G. Jannink, Polymers in Solution: Their Modelling and Structure (Oxford Univ. Press: NY, 2010).

[27] M. Müller and K. Daoulas, J. Chem. Phys. 128024903 (2008).

[28] Y. Deng and B. Roux, J. Chem. Phys. 128115103 (2008).

[29] K.A. Connors, Chemical Kinetics, (VCH Publishers, 1990).

[30] S. Harrington, R. Zhang, P.H. Poole, F. Sciortino, and H.E. Stanley, Phys. Rev. Lett, 782409 (1997).

[31] L.D. Landau and E.M. Lifshitz, Statistical Physics 3rd edition (Pergamon: Oxford, 1981).

[32] M.V. Tamm, A.B. Shkarin, V.A. Avetisov, O.V. Valba, and S.K. Nechaev, Supplementary online materials to this paper.

[33] V.A. Avetisov et al., in preparation.

[34] D. Strauss, SIAM Review, 28, 513 (1986).

[35] J. Park, and M.E.J. Newman, Phys. Rev. E, 72, 026136 (2005). 
[36] A.G. Chandrasekhar and M.O. Jackson, arXiv.org: 1210.7375 .

[37] M. Eigen, Naturwissenschaften 58465 (1971).

[38] S. Galluccio, R. Graber, and Y.-C. Zhang, J.Phys. A: Math. Gen. 29 L249 (1996).

[39] L. Peliti, Europhys. Lett. 57745 (2002).

[40] M. Kolár and F. Slanina, Physica A, 313549 (2002).

[41] E.A. Bender, and E.R. Canfield, Journal of Combinatorial Theory A, 24, 296 (1978).
[42] In 22] the authors use renormalized densities of the motifs. In our notation, the components of their motif vector $\mathbf{Z}$ are equal to $Z_{i}=\left(c_{i}-c_{i}^{0}\right) / \chi_{i}=\Delta c_{i} / \chi_{i}$, where $c_{i}^{0}=c_{i}(h=0)$, and $\chi_{i}$ is the susceptibility of $i$ th concentration to an external field $h_{i}$ coupled to it, taken at $h_{i}=0$. This linear shift does not change the results presented in our work. 